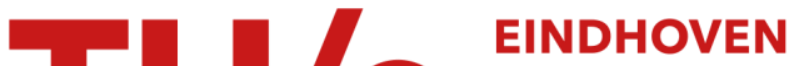 UNIVERSITY OF TECHNOLOGY
}

\section{Mimo pillow : an intelligent cushion designed with maternal heart beat vibrations for comforting newborn infants}

\section{Citation for published version (APA):}

Chen, W., Bambang Oetomo, S., Tetteroo, D., Versteegh, F., Mamagkaki, T., Serras Pereira, M., Janssen, L., \& Meurs, van, A. (2015). Mimo pillow : an intelligent cushion designed with maternal heart beat vibrations for comforting newborn infants. IEEE Journal of Biomedical and Health Informatics, 19(3), 979-985. https://doi.org/10.1109/JBHI.2014.2349153

DOI:

10.1109/JBHI.2014.2349153

Document status and date:

Published: 01/01/2015

\section{Document Version:}

Accepted manuscript including changes made at the peer-review stage

\section{Please check the document version of this publication:}

- A submitted manuscript is the version of the article upon submission and before peer-review. There can be important differences between the submitted version and the official published version of record. People interested in the research are advised to contact the author for the final version of the publication, or visit the $\mathrm{DOI}$ to the publisher's website.

- The final author version and the galley proof are versions of the publication after peer review.

- The final published version features the final layout of the paper including the volume, issue and page numbers.

Link to publication

\section{General rights}

Copyright and moral rights for the publications made accessible in the public portal are retained by the authors and/or other copyright owners and it is a condition of accessing publications that users recognise and abide by the legal requirements associated with these rights.

- Users may download and print one copy of any publication from the public portal for the purpose of private study or research.

- You may not further distribute the material or use it for any profit-making activity or commercial gain

- You may freely distribute the URL identifying the publication in the public portal.

If the publication is distributed under the terms of Article 25fa of the Dutch Copyright Act, indicated by the "Taverne" license above, please follow below link for the End User Agreement:

www.tue.nl/taverne

Take down policy

If you believe that this document breaches copyright please contact us at:

openaccess@tue.nl

providing details and we will investigate your claim. 


\title{
Mimo Pillow - an Intelligent Cushion Designed with Maternal Heart Beat Vibrations for Comforting Newborn Infants
}

\author{
Wei Chen, Senior Member, IEEE, Sidarto Bambang Oetomo, MD, Daniel Tetteroo, Frank Versteegh, \\ Thelxi Mamagkaki, Mariana Serras Pereira, Lindy Janssen, Andrea van Meurs
}

\begin{abstract}
Premature infants are subject to numerous interventions ranging from a simple diaper change to surgery while residing in Neonatal Intensive Care Units (NICUs). These neonates often suffer from pain, distress and discomfort during the first weeks of their lives. Although pharmacological pain treatment often is available, it cannot always be applied to relieve a neonate from pain or discomfort. This paper describes a non-pharmacological solution, called Mimo, which provides comfort through mediation of a parent's physiological features to the distressed neonate via an intelligent pillow system embedded with sensing and actuating functions. We present the design, the implementation and the evaluation of the prototype. Clinical tests at Máxima Medical Centre in the Netherlands show that among the 9 of 10 infants who showed discomfort following diaper change, a shorter recovery time to baseline Skin Conductance Analgesimeter (SCA) values could be measured when the maternal heartbeat vibration in the Mimo was switched on and in 7 of these 10 a shorter crying time was measured.
\end{abstract}

Index Terms - preterm neonates, comforting solution, bonding, heartbeat, user-centered design

\section{INTRODUCTION}

Babies that are born after a pregnancy lasting 37 weeks or less are typically considered premature. Critically ill neonates are a special group of patients that consist of premature infants who may suffer from diseases that are mainly caused by immaturity of their organs, and full term infants, who become severely ill during or immediately after birth. Critically ill newborn infants are normally admitted to a Neonatal Intensive

W. Chen and D. Tetteroo are with the Department of Industrial Design, Eindhoven University of Technology, 5612 AZ, Eindhoven, The Netherlands (e-mail: w.chen@tue.nl; d.tetteroo@ tue.nl).

S. Bambang Oetomo is with the Neonatal Intensive Care Unit, Department of Pediatrics, Máxima Medical Center, 5500 MB, Veldhoven, The Netherlands, and the Department of Industrial Design, Eindhoven University of Technology, 5612 AZ, Eindhoven, The Netherlands (e-mail: s.bambangoetomo@mmc.nl).

Frank Versteegh, Thelxi Mamagkaki, Mariana Serras Pereira were with the PDEng program on User System Interaction (USI), Department of Industrial Design, Eindhoven University of Technology, 5612 AZ, Eindhoven. Mariana Serras Pereira is with the Tilburg Center for Cognition and Communication (TICC), Tilburg University, The Netherlands. (email: profiler@gmail.com; thelxi@gmail.com; m.serraspereira@uvt.nl ).

Lindy Janssen and Andrea van Meurs were with the Neonatal Intensive Care Unit, Department of Pediatrics, Máxima Medical Center, 5500 MB, Veldhoven, The Netherlands. Lindy Janssen works at the Neonatal Intensive Care Unit of the Radboud UMC, Nijmegen, The Netherlands. Andrea van Meurs is a medical student at the University of Utrecht, The Netherlands. (e-mail: lindy.janssen@ radboudumc.nl; a.vanmeurs@ students.uu.nl)
Care Unit (NICU) for treatment by neonatologists and specialized nurses. Survival of the preterm neonates is unfortunately reliant on many painful medical interventions. These interventions include, but are not limited to: heel punctures, venepunctures and in- or detubations [1]. The attachment and removal of sensors required for measuring physiological signals can also be unpleasant, stressful and painful [2]. Although pain treatment is given in some of these cases, a large proportion $(50 \%)[1,3]$ of the interventions are performed without any form of pain treatment.

Simple procedures and environmental factors are also possible sources of pain and discomfort to preterm neonates. Nociception is the term used to refer to the neural processes of encoding and processing painful stimuli. In preterm neonates this nociceptional system is not yet fully developed. A possible consequence of this not-fully developed nociceptional system is that stimuli such as sound and light, which are normally not considered painful to a human being, may be perceived as painful by the preterm neonate [4]. Injuries, but also clinical procedures, will result in more widespread sensitivity to pain [5]. The stay of a preterm infant in the incubator is negatively influenced by stress (excessive light and noise) and painful procedures [6]. Exposure to repetitive pain, stress and discomfort in premature infants is associated with cognitive and neuro developmental impairments [7]. The consequences of untreated procedural pain in neonates can be both severe and long term. It is believed that exposing preterm neonates to pain negatively affects their ability to cope with pain and stress in later childhood [5,8]. There is growing awareness that pain following a painful procedure should be treated appropriately and adequate soothing by parents or nurses is advocated.

Neonates show many different responses to pain and stress. These responses can be of both physiological and behavioural nature, and can be used to detect discomfort of a neonate. Physiological responses include an increased heart and respiratory rate, increased or decreased blood pressure, decreased oxygen saturation, vagal tone and skin temperature. Behavioural responses are even more varied. Examples include vocalizations like crying and whimpering, facial expressions like grimacing with eyes squeezed shut or a stretched open mouth, and body movements like finger and toe splaying and trunk arching $[5,9]$. It is worth noting that a change in any of these responses does not always imply that the neonate is experiencing stress or discomfort. Several pain-scoring systems 
(such as PIPP [10] and Comfort-NEO [11]) have been developed to aggregate the behavioural signs, providing a reliable and structured tool for pain measurement.

The past two decades have seen an increase in interest in neonatal pain treatment [8]. Pharmacological solutions remain popular and are the most used method for pain treatment. Non-pharmacological methods of providing comfort and pain relief do exist and some have proven to be effective. The most popular techniques include: non-nutritive sucking, offering a sweet solution, facilitated tucking and skin-to-skin care.

Non-nutritive sucking was one of the first non-pharmacological comforting techniques to be studied and refers to offering a pacifier for the neonate to suck on [12]. A sucrose solution can be provided as an extra means of comfort during more painful procedures. A syringe is used to gently squeeze some sucrose on the inside of the neonate's cheek. A pacifier is offered after the delivery of the sucrose, distracting the infant with something sweet to suck on [13].

During facilitated tucking, the caregiver holds one hand on the baby's head and another one on the feet or body, depending on what is feasible at that moment [14]. Similar techniques are containment and swaddling [15]. The aim of all these techniques is to restrict the movement space for the neonate and to maintain it in a position similar to in utero.

One of the most successful comforting techniques is skin-to-skin care (SSC), otherwise known as kangaroo mother care [16]. During SSC the mother and infant are wrapped in cloth together, with the infant held to the bare chest of the mother. One downside of this comforting technique is that the neonate needs to be taken out of the incubator, which can be stressful to the child. Some neonates are unable to receive this particular kind of care, because their medical condition does not allow them to be taken out of the incubator.

Other comforting techniques include presenting the neonate with an audible heartbeat [17] or music [18] and aromatherapy [19]. If possible, breastfeeding can also be used as a non-pharmacological comforting technique, although its effect was demonstrated on term neonates [20].

The majority of these comforting techniques have in common that a parent or nurse needs to be present to provide the desired care. Although the sole presence of parents might be comforting to a neonate, most parents are unable to be with their child continuously. The same holds for nurses, as they often have multiple neonates to care for. A comforting solution that does not require the continued presence of a parent or nurse is thus a design path worth exploring.

The Eindhoven University of Technology (TU/e) has started a 10-year project on perinatology research in cooperation with the Máxima Medical Centre (MMC) in the Netherlands. The goal of this project is to improve the wellbeing for the pregnant woman and her child before, during and after delivery [21-23]. Research results that have been reported from this project area, for example, a smart jacket for neonates integrated with textile sensors [24, 25], a power supply based on contactless energy transfer [26], blood oxygen saturation monitoring for neonates using a reflectance pulse oximeter [27, 28], neonatal temperature monitoring [29], textile mat design [30], data transmission [31, 32], and a device to support cardiopulmonary resuscitation of neonates [33].

This paper presents a new concept and design for comforting preterm neonates, which aims at comforting preterm neonates by mimicking selected features of the baby's mother or father and making the features available to the baby when the parents cannot be present themselves. We were inspired by the fact that kangaroo mother care (KMC) is beneficial for newborn infants. Apart from the skin to skin contact and maternal scent, one of the features of KMC is the exposure to the maternal heart beat [34]. Doheny et al. [35] reported that exposure to biological maternal sounds improves cardiorespiratory regulation in extremely preterm infants. Although the effect of a mother's heartbeat vs. any other heartbeat has not been shown, it is beneficial to use a parental heartbeat. Empowering parents to play an active role in their child's incubation process is very important [36]. By letting parents 'donate' their heartbeat to the child, they feel that they can contribute to the child's wellbeing which is an important factor in the parent child bonding process [37, 38]. The concept, called Mimo, is a comforting solution through an intelligent pillow system embedded with sensing and actuating functions that provides neonates with an experience similar to being held by their parents. The following sections will present the concept in more detail, and describe the implementation and evaluation of the prototype at Máxima Medical Centre in the Netherlands.

\section{DESIGN CONCEPT}

The concept for the proposed comforting solution was based on findings from literature and the user studies performed at NICU.

\section{A. User studies}

An essential first step in the development of a new, non-pharmacological pain treatment is the recognition and evaluation of the status-quo. We apply User Centered Design (UCD) during the industrial design process and based on the outcome of user research define system requirements and make decision on technological directions. In UCD the end-user is constantly involved in the design process. In this case, medical staff and parents can be valuable sources of information, in effect becoming the voice of the neonates. Interviews with medical staff and observations at a NICU facility have been performed in order to gain better insight on the conditions and treatments given at a NICU.

Neonates can be comforted in two ways: by providing an active comforting treatment, or by taking away sources of discomfort. Some hospitals (e.g., the Maternity District Hospital "Helena Venizelou" (GR)) have a very strict policy that focuses on the latter. The fragile neonates are taken out of the incubator only for medical treatments. The care policy focuses on limiting external stimuli to the neonates as much as possible. Noise and light levels are reduced to absolute minimum and parents can visit their babies during fixed visiting hours. Other hospitals (e.g., MMC in Veldhoven (NL) and the Maastricht University Medical Centre (NL)) are less strict and emphasize on the value of parent-child bonding as a source of 
active comfort, therefore they allow parents to visit their baby in the NICU at any given time. These hospitals also encourage the parents to take part in their child's care, by changing diapers or bottle feeding. This kind of involvement also helps the parents who often feel that they cannot help their fragile baby during the stay at the NICU. These hospitals encourage kangaroo mother care as a means of comfort and bonding. Nurses and parents from these hospitals stated that infants are the most relaxed during kangaroo mother care.

Although it is clear that parents can play an important role in the comfort of their child, they cannot be present at all times to provide this comfort. In all hospitals, some more than others, there are situations in which alternative means of comforting are required. Therefore, it seems appropriate that a new comforting solution would focus on providing a neonate with the same feeling of comfort that parents would provide, even when the parents cannot be physically present.

\section{B. Concept}

Based on the literature review, the aforementioned user studies and a brainstorm, a concept for a novel solution of comforting preterm neonates were proposed. This concept, called Mimo, is a comforting solution that provides neonates with an experience similar to being held by their parents.

Since neonates show less stress signs when they are in contact with their parents and held to their chest, which seems to be one of the most pleasant experiences for a preterm neonate, Mimo aims at providing a comforting solution with qualities similar to those of being held to a parent's chest, available especially when the parent cannot provide the comfort him- or herself. This concept might not only increase the general comfort level, but it can also contribute to the bonding between parent and infant. Mimo gives parents the chance to record their personal features such as heartbeat, smell and temperature, which can be mediated to the child when it is in need of comfort. When the concept is fully realized, the child might experience a feeling as the parents were close to them, even when the parents are not physically there. One of Mimo's basic characteristics is its ease of use, which enables parents to record their physiological features without guidance.

\section{PROTOTYPE}

The initial prototype of Mimo was designed to mediate the parent's heartbeat by means of vibrations, much like a pulse.

One of the basic characteristics of the concept is that Mimo should be easy to set up and use, so that parents could record their physiological data on their own, without the guidance of a nurse for example. Then, the nurse needs only to switch on and activate Mimo when comforting is needed. Moreover, the Mimo prototype features a pillow-like device to convey the heartbeat, which can replace the regular pillows that are used in incubators, thus sustaining the neonate's environment and avoiding extra special equipment that would load the incubator. This prototype was designed to be comfortable and ergonomic for a premature neonate, but also washable and clinically safe to meet hospital standards.

Options for reproducing heartbeats both in terms of sensing and output/actuation have already been explored [17]. However, we strived to develop a system that would be very easy to use without the need for medical personnel assisting in the process. The PPG (Photoplethysmogram) sensor is non-invasive and easy to use by the parents. Moreover, the main objective of our research is to develop a device that would provide comfort both to the child and the parents by empowering parents to make active contribution even when they are not present to their baby. Therefore, we designed the system to measure PPG signals and play heart beat vibrations to comfort the baby. A study on an intelligent baby carriage with a PPG system and heart beat vibration feedback has been reported in [39] with preliminary experimental evaluation of only one child. In our study we run clinical tests to investigate the effectiveness of Mimo.

The Mimo prototype (see Fig. 1) consists of two parts:

1) A recording unit (Mimo Recorder), for registration of the parent's heartbeat

2) A pillow-like object (Mimo Pillow), for transmitting the parent's heartbeat to the neonate.

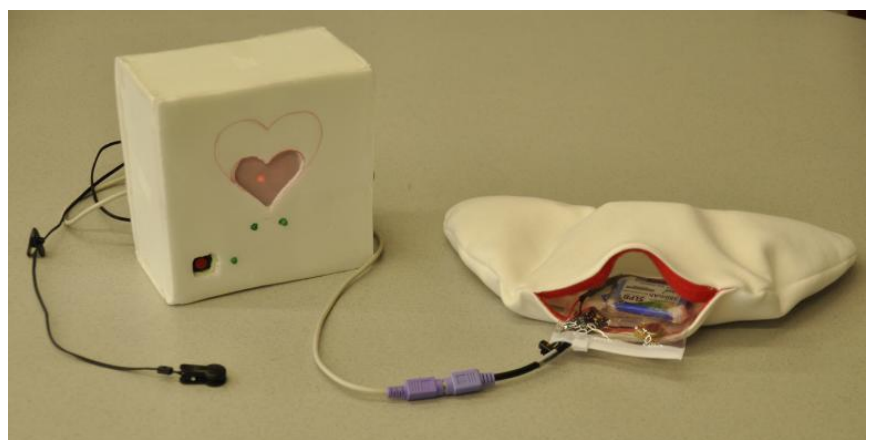

Fig. 1. Prototype with the Mimo Recorder (left) and the Mimo Pillow (right).

\section{B. Mimo Recorder}

The Mimo Recorder is a white, cubical box that allows parents of preterm neonates to make a recording of their heartbeat. A heartbeat photoplethysmograph (PPG) sensor, as well as a connection cable are attached to the side of the box. The front of the box features a number of LEDs displaying the status of the recording process and a button to initiate the recording process.

\section{1) Sensor}

The heartbeat sensor that is used in the prototype is a PPG sensor, which is an optical sensor that measures a person's pulse. An infra-red LED on one side of the sensor shines through the finger onto a photosensitive sensor at the other side of the finger. The amount of blood that flows through the finger influences the amount of light that reaches the sensor, hence providing information about the person's pulse. Although there are other ways to record a heartbeat (such as electrocardiogram sensors), the PPG sensor provides a quick and easy way to record a heartbeat for this application.

\section{2) Electronics}

An overview of the circuitry inside the Mimo Recorder can be found in Fig. 2. The heart of the Mimo Recorder is an Arduino Uno microcomputer, powered by a $9 \mathrm{~V}$ battery. The Arduino handles almost the complete recording process, 
ranging from sensing and storage to transmission of the heartbeat to the Pillow. It was deliberately chosen to use a battery rather than a fixed power supply, to provide maximum flexibility to parents in their choice of a recording location.

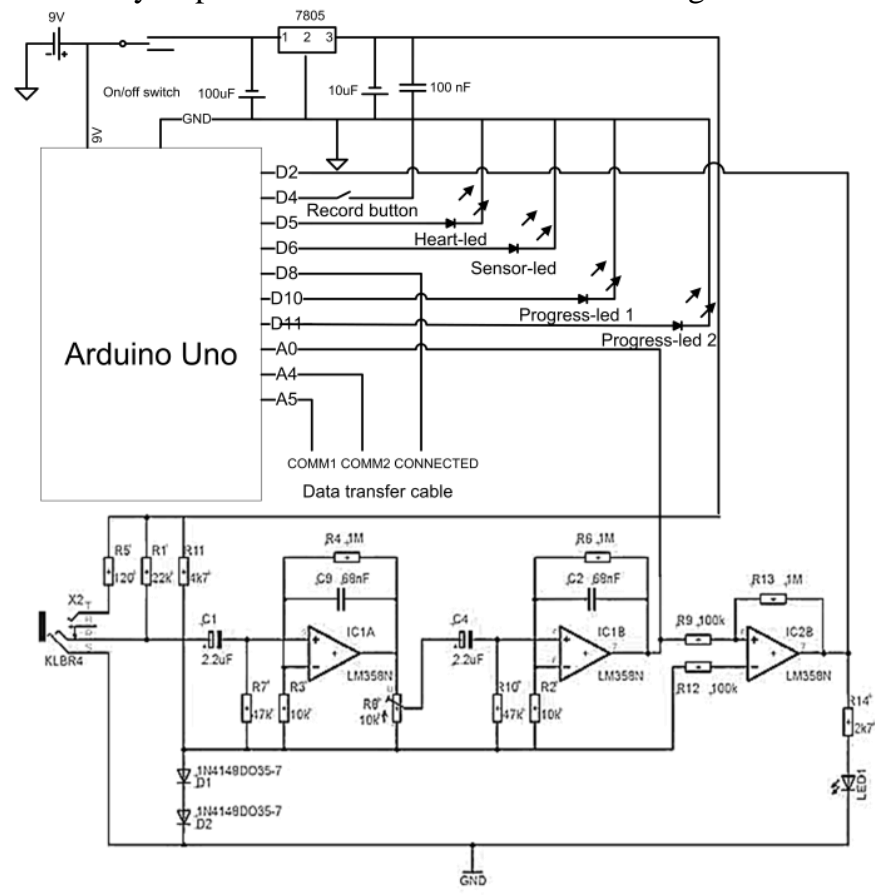

Fig. 2. Circuit diagram of the electronics in the Mimo Recorder.

\section{3) User Interface}

The user interface of the Mimo Recorder was designed to be as simple as possible, so that parents can perform the complete recording process themselves. Four LEDs provide feedback to the parents about the status of the heartbeat recording. One light shows whether the device is switched on, another light indicates whether the sensor is properly attached and the two remaining lights indicate whether the recording has not started yet (lights off), is currently in progress (lights blinking) or has finished (lights on).

In order to record their heartbeat, parents first need to attach the sensor to their finger or earlobe. Once the sensor indicator confirms that the sensor has been properly attached, the parent can press the recording button and wait for the status lights to light steady. Then the pillow can be connected to the recording unit and the heartbeat will be transferred to the pillow.

\section{Mimo Pillow}

The Mimo Pillow is the part of the prototype that stays with the neonate. It is a boomerang-shaped pillow that is filled with polymer beads and contains a pouch that accommodates the electronics. The polymer beads that are used for the filling act as support and ensure both softness for the neonate as well as stability that allow the pillow to be shaped around the neonate's body. The form of the pillow was determined through an iterative design process - inspired by the design of the 'Frederick T. Frog' pillow by Philips [40], and matured through discussions with medical staff. The materials were chosen on the basis of their suitability for the NICU environment (safe, easy to clean, comfortable for the child).

\section{1) Electronics}

Much like the Mimo Recorder, the Mimo Pillow is controlled by an Arduino Mini microcomputer. This Arduino version is much smaller and flatter than the one used in the Mimo Recorder, such that it is hardly felt from the outside of the pillow. Other efforts to ensure that electronics are not felt by the neonate have been made by using a specialized flat model racing battery as power supply, a tiny vibration motor to mediate the heartbeat vibrations, and by using a tiny control to switch on or off the electronics. A detailed overview of the electronic circuit is provided in Fig. 3.

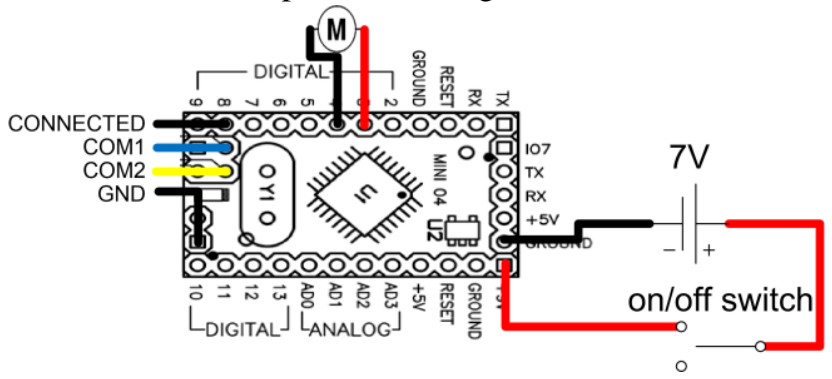

Fig. 3. Circuit diagram of the electronics in the Mimo Pillow.

\section{2) User Interface}

The heartbeat data that has been recorded with the Mimo Recorder can be transferred to the Mimo Pillow by connecting the connection cables of both devices. Once the heartbeat data has been transferred to the pillow, it is immediately ready for use. The recorded heartbeat data is stored in the microcomputer in the Mimo Pillow and is played back continuously, as long as the device is switched on.

\section{Clinical tests And evaluation}

To test the viability of the design, clinical tests were carried out at Máxima Medical Center in the Netherlands. The objective is to assess the effects of the Mimo system on newborn infants following a discomforting procedure. We hypothesized that sensing of the maternal heart beat by the infant could offer additional consolation and hence reduce the crying time following a discomforting procedure. An analysis of risks was performed before applying the prototype to the NICU. The safety of the Mimo prototype system was analyzed by clinical physicists and the ethical commission of the MMC approved the experiments.

\section{A. Method}

First, the heartbeats of the neonates' mothers were recorded with the Mimo Recorder. The signal was uploaded to the Mimo pillow and after switching on the rhythmic vibrations can be felt at the surface of the pillow. The Mimo pillow was placed on the chest of the infants after their diaper change before their feeding, a moment of discomfort where most baby's cry. Fig. 4 shows that a mother records her heart beat at NICU using the Mimo recorder and a baby with the Mimo pillow during one test. To rule out any effects of consolation by the pillow without heart beat vibrations, we tested the pillow both with the vibrations switched on and the vibrations switched off during two sub sequential diaper changes. 


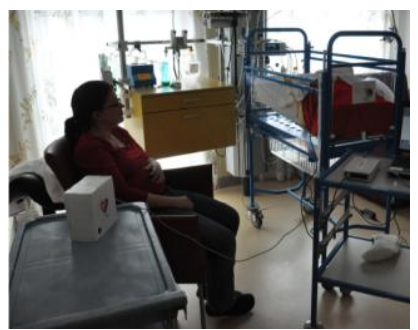

(a)

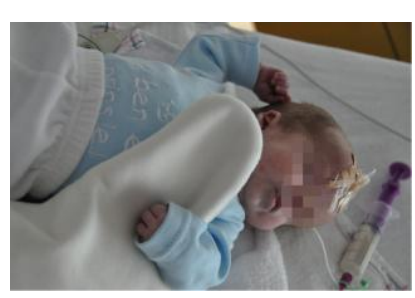

(b)
Fig. 4. (a) A mother records her heart beat at NICU using the Mimo recorder. (b) A baby with the Mimo pillow during the test.

We conducted a pilot study in late preterm infants that were admitted at the medium care ward of Máxima Medical Center. Patients were randomly assigned to 1) comforting with the pillow with vibrations switched on, followed by comforting with the vibrations switched off and 2) comforting with the vibrations switched off, followed by comforting with the pillow with vibrations switched on. The change in comfort level following placement of the pillow on the infants chest was assessed by video recording of the facial expressions to measure crying time and by the measurement of recovery time to baseline values using the Skin Conductance Analgesimeter (SCA) [41]. The mean baseline SCA value was measured for each baby when he or she was in a calm condition before the diaper change. During the diaper change, the SCA values increased due to the discomfort of neonates. The recovery time was calculated by measuring the duration of time after the diaper change for the SCA values returning to the baseline SCA value. The observer (AvM) was unaware of the vibrations in the Mimo pillow being switched on or off.

\section{B. Results}

19 infants with a gestational age between $32+7$ and $36+6$ weeks enrolled in the study. Nine infants did not show any sign of discomfort after the diaper change and obviously did not show any change in study parameters following placement of the Mimo pillow on the chest of the infants. The results of the other 10 infants that cried after the diaper change are shown in Table 1.

In this study we show that 9 of 10 infants that showed discomfort following diaper change, a shorter recovery time to baseline SCA values could be measured when the maternal heartbeat vibration in the Mimo was switched on. In 7 of these 10 a shorter crying time was measured. Differences between mimo-off and mimo-on, have been tested by the Wilcoxon
Rank Sum test yielding a p-value of 0.037 in recovery time and 0.086 in crying time respectively. The results indicate that preterm infants can benefit from the Mimo pillow during and following stressful procedures. The device seems to be a simple, effective and safe intervention for pain in newborn infants.

\section{DISCUSSION}

The Mimo concept consists of two parts: a recording unit and a technologically enhanced pillow. The recording unit is used to record physiological data (such as heartbeat, breathing rhythm, parental smell and body temperature) from a parent. This data can then be transferred to the pillow, which includes technology that enables it to 'replay' the recorded data to the neonate. The current prototype achieves a part of the functions of the original concept, as it focuses only on the recording and playback of a parent's heartbeat. In the future work, the complete functions for more sensing and actuating modalities (e.g. breathing rhythm, parental smell and body temperature) will be developed. Further developments also include miniaturization of circuits and electronics and a Flash disk or wireless module (e.g. Bluetooth) to store / transfer the mother heart beat data to the pillow.

The parent-child bonding is loose for the NICU patients because of the separation of neonates from their parents. The use of Mimo is expected to comfort the neonates on one hand and at the meanwhile to comfort parents and help them feel much closer to their babies. We carried out preliminary interviews with parents at MMC and online by demonstrating them the use of Mimo. The feedback from parents show that Mimo plays a positive role in comforting parents and attaining parental role by helping them feel much closer to their babies and feel that they are always there to support their babies.

More clinical tests will be carried out in the future study to compare Mimo with other neonatal pain treatments (e.g. sucking or swaddling) and compare the effectiveness of parental heart beat with any other heart beat in order to further investigate the analgesic effect of the heartbeat of mother.

The crying time was assessed by observation on the babies' facial expression. Because the participants are premature infants and their facial expression is not as well developed as the facial expression of a term infant, the physiological measure of recovery time to baseline SCA value is more reliable than observation. More physiological data, such as ECG, EEG, and heart rate variability, and behavioral data of the neonates could

TABLE 1: THE EFFECTS OF THE MIMO PILLOW ON NEWBORN INFANTS FOLLOWING A DISCOMFORTING PROCEDURE.

\begin{tabular}{|c|c|c|c|c|c|c|}
\hline \multirow[t]{2}{*}{$\begin{array}{c}\text { Infant } \\
\#\end{array}$} & \multirow[t]{2}{*}{$\begin{array}{l}\text { Gest. age } \\
\text { (wk) }\end{array}$} & \multirow[t]{2}{*}{$\begin{array}{l}\text { Post natal age } \\
\text { (days) }\end{array}$} & \multicolumn{2}{|c|}{$\begin{array}{l}\text { Recovery time to baseline } \\
\text { SCA value (sec) }\end{array}$} & \multicolumn{2}{|c|}{$\begin{array}{l}\text { Crying time } \\
(\mathrm{sec})\end{array}$} \\
\hline & & & Mimo on & Mimo off & Mimo on & Mimo off \\
\hline 1 & $34+5$ & 13 & 2 & 8 & 19 & 228 \\
\hline 2 & $34+5$ & 1 & 6 & 14 & 23 & 44 \\
\hline 3 & $34+5$ & 3 & 2 & 6 & 11 & 37 \\
\hline 4 & $37+4$ & 11 & 10 & $>300$ & 38 & 279 \\
\hline 5 & $35+3$ & 2 & 6 & 12 & 34 & 49 \\
\hline 6 & $35+0$ & 11 & 32 & 4 & 36 & 36 \\
\hline 7 & $34+3$ & 3 & 2 & 4 & 18 & 31 \\
\hline 8 & $32+6$ & 30 & 64 & 112 & 63 & 34 \\
\hline 9 & $35+0$ & 12 & 26 & 146 & 55 & 52 \\
\hline 10 & $33+4$ & 12 & 6 & 16 & 31 & 58 \\
\hline
\end{tabular}


also be measured for the purpose of multi-modal assessment and statistical analysis.

Furthermore, the current design is only a prototype for tests and demonstration purposes. To develop the solution to a ready-to-market product, strict product design process and clinical safety evaluation of the reliability of the complete system are needed.

\section{CONCLUSION}

This paper presents the design, implementation and evaluation of a new, non-pharmacological comforting solution for preterm neonates. The concept, named Mimo, aims at comforting a neonate by providing the baby with a feeling similar to that of being close to a parent. An intelligent pillow system was proposed and implemented with embedding sensing and actuating functions for the transfer of maternal heart beat into the pillow and playing the pulsation to the neonate. The prototype was tested on premature babies at Máxima Medical Centre in the Netherlands. Among the 9 of 10 infants who showed discomfort following diaper change, a shorter recovery time to baseline Skin Conductance Analgesimeter (SCA) values could be measured when the maternal heartbeat vibration in the Mimo was switched on and in 7 of these 10 a shorter crying time was measured. Thus this device has potential to be a simple, effective and safe intervention for comforting newborn infants.

\section{ACKNOWLEDGEMENTS}

We are thankful for the great help received from Ms. Astrid Osagiator, the senior NICU nurse of Máxima Medical Center in the Netherlands, as well as the parents and neonates that participated in this study.

\section{REFERENCES}

[1] R. Carbajal, "Nonpharmacological Treatment of Neonatal Pain," in Neonatal Pain, G. Buonocore and C. Bellieni, Eds., ed: Springer Milan, 2008, pp. 83-97.

[2] D. Barker and N. Rutter, "Exposure to invasive procedures in neonatal intensive care unit admissions," Archives of Disease in Childhood-Fetal and Neonatal Edition, vol. 72, pp. F47-F48, 1995.

[3] C. C. Johnston, et al., "Kangaroo mother care diminishes pain from heel lance in very preterm neonates: a crossover trial," $B M C$ pediatrics, vol. 8, p. 13, 2008.

[4] K. Anand, "Consensus statement for the prevention and management of pain in the newborn," Archives of pediatrics \& adolescent medicine, vol. 155, pp. 173-180, 2001.

[5] M. I. Levene, "Minimizing the discomfort of neonatal intensive care," Current Paediatrics, vol. 13, pp. 196-200, 2003.

[6] A. T. Bhutta and K. J. Anand, "Vulnerability of the developing brain. Neuronal mechanisms," Clin Perinatol, vol. 29, pp. 357-372, 2002.

[7] R. E. Grunau, et al., "Neonatal pain, parenting stress and interaction, in relation to cognitive and motor development at 8 and 18 months in preterm infants," Pain, vol. 143, pp. 138-146, 2009.

[8] C. C. Johnston, et al., "Pain in neonates is different," Pain, vol. 152, pp. S65-S73, 2011.

[9] D. Ballweg, "Neonatal and pediatric pain management: standards and application," Paediatrics and Child Health, vol. 18, pp. S61-S66, 2008.

[10] B. Stevens, et al., "Premature Infant Pain Profile: development and initial validation," The Clinical journal of pain, vol. 12, pp. 13-22, 1996.
[11] M. van Dijk, et al., "Taking up the challenge of measuring prolonged pain in (premature) neonates: the COMFORTneo scale seems promising," The Clinical journal of pain, vol. 25, pp. 607-616, 2009.

[12] S.-Y. P. K. Shiao, et al., "Meta-analysis of the effects of nonnutritive sucking on heart rate and peripheral oxygenation: research from the past 30 years," Issues in Comprehensive Pediatric Nursing, vol. 20, pp. 11-24, 1997.

[13] B. Stevens, et al., "Sucrose for analgesia in newborn infants undergoing painful procedures," Cochrane Database of Systematic Reviews, 2013.

[14] A. Axelin, et al., "Facilitated tucking by parents' in pain management of preterm infants - a randomized crossover trial," Early human development, vol. 82, pp. 241-247, 2006.

[15] C.-M. Huang, et al., "Comparison of pain responses of premature infants to the heelstick between containment and swaddling," Journal of Nursing Research, vol. 12, pp. 31-35, 2004.

[16] T. C. Castral, et al., "The effects of skin-to-skin contact during acute pain in preterm newborns," European Journal of Pain, vol. 12, pp. 464-471, 2008.

[17] H. Kurihara, et al., "Behavioral and adrenocortical responses to stress in neonates and the stabilizing effects of maternal heartbeat on them," Early human development, vol. 46, pp. 117-127, 1996.

[18] M. Butt and B. Kisilevsky, "Music modulates behaviour of premature infants following heel lance," The Canadian journal of nursing research $=$ Revue canadienne de recherche en sciences infirmières, vol. 31, pp. 17-39, 2000.

[19] R. M. Sullivan and P. Toubas, "Clinical usefulness of maternal odor in newborns: soothing and feeding preparatory responses," Biology of the Neonate, vol. 74, pp. 402-408, 1998.

[20] R. Carbajal, et al., "Analgesic effect of breast feeding in term neonates: randomised controlled trial," BMJ: British Medical Journal, vol. 326, p. 13, 2003.

[21] W. Chen, et al., "Neonatal Monitoring: Current Practice and Future Trends," in Handbook of Research on Developments in E-Health and Telemedicine: Technological and Social Perspectives, ed: IGI Global, 2010, pp. 939-961.

[22] W. Chen, et al., Neonatal Monitoring Technologies: Design for Integrated Solutions: IGI Global, Hershey, PA, USA, 2012.

[23] W. Chen, et al., "Sensor integration for perinatology research," International Journal of Sensor Networks, vol. 9, pp. 38-49, 2011.

[24] S. Bouwstra, et al., "Smart jacket design for neonatal monitoring with wearable sensors," in Sixth International Workshop on Wearable and Implantable Body Sensor Networks (BSN 2009) 2009, pp. 162-167.

[25] F. P. Landete, et al., "Context Aware Sensing for Health Monitoring," in the 34th Annual International IEEE EMBS Conference of the IEEE Engineering in Medicine and Biology Society (EMBC), 2012, pp. 1294-1297.

[26] W. Chen, et al., "A design of power supply for neonatal monitoring with wearable sensors," Journal of Ambient Intelligence and Smart Environments, vol. 1, pp. 185-196, 2009.

[27] W. Chen, et al., "Non-invasive blood oxygen saturation monitoring for neonates using reflectance pulse oximeter," in Proceedings of the Conference on Design, Automation and Test in Europe, 2010, pp. 1530-1535.

[28] D. Potuzakova, et al., "Innovative Design for Monitoring of Neonates Using Reflectance Pulse Oximeter," in 7th International Conference on Intelligent Environments (IE2011) 2011, pp. 200-205.

[29] W. Chen, et al., "Monitoring body temperature of newborn infants at neonatal intensive care units using wearable sensors," in Proceedings of the Fifth International Conference on Body Area Networks, 2010, pp. 188-194.

[30] R. van Donselaar and W. Chen, "Design of a smart textile mat to study pressure distribution on multiple foam material configurations," in Proceedings of the 4th International Symposium on Applied Sciences in Biomedical and Communication Technologies, 2011, p. 129.

[31] W. Chen, et al., "Wireless transmission design for health monitoring at neonatal intensive care units," in 2nd International Symposium on Applied Sciences in Biomedical and Communication Technologies (ISABEL 2009) 2009, pp. 1-6.

[32] W. Chen, et al., "Design of Wireless Sensor System for Neonatal Monitoring," in the third international workshop on Wireless 
Sensor Networks: theory and practice, in conjunction with the 4th IFIP International Conference on New Technologies, Mobility and Security (NTMS 2011) 2011, pp. 1-5.

[33] W. Chen, et al., "Rhythm of Life Aid (ROLA): an integrated sensor system for supporting medical staff during cardiopulmonary resuscitation (CPR) of newborn infants," Information Technology in Biomedicine, IEEE Transactions on, vol. 14, pp. 1468-1474, 2010.

[34] L. Gray, et al., "Skin-to-skin contact is analgesic in healthy newborns," Pediatrics, vol. 105, pp. e14-e14, 2000.

[35] L. Doheny, et al., "Exposure to biological maternal sounds improves cardiorespiratory regulation in extremely preterm infants," Journal of Maternal-Fetal and Neonatal Medicine, vol. 25, pp. 1591-1594, 2012.

[36] P. W. Fowlie and H. McHaffie, "ABC of preterm birth: Supporting parents in the neonatal unit," BMJ: British Medical Journal, vol. 329, p. 1336, 2004.

[37] L. Fegran, et al., "A comparison of mothers' and fathers' experiences of the attachment process in a neonatal intensive care unit," Journal of Clinical Nursing, vol. 17, pp. 810-816, 2008.

[38] M. Bialoskurski, et al., "The nature of attachment in a neonatal intensive care unit," The Journal of perinatal \& neonatal nursing, vol. 13, pp. 66-77, 1999.

[39] T. Kawashima, "Study on intelligent baby carriage with power assist system and comfortable basket," Journal of mechanical science and technology, vol. 23, pp. 974-979, 2009. Frederick $T . \quad$ Frog, available from:
http://www.healthcare.philips.com/main/products/mother_and_chi ld_care/post_natal_care/nicu/sleep_and_positioning/freddy/.

[41] H. Storm, "Skin conductance and the stress response from heel stick in preterm infants," Archives of Disease in Childhood-Fetal and Neonatal Edition, vol. 83, pp. F143-F147, 2000.

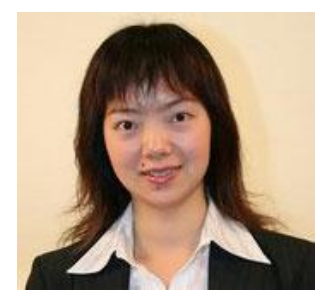

Wei Chen (M'07, SM'12) received her B. Eng. degree in 1999 and M. Eng. degree in 2002 on telecommunication systems and smart sensor systems from School of Electronics and Information Engineering, Xian Jiaotong University, China. She obtained her Ph.D. degree in 2007 on performance monitoring and impairment mitigation for optical communication systems, from the Department of Electrical \& Electronics Engineering, The University of Melbourne, Australia. She worked at Bell Laboratories, Alcatel-Lucent, Stuttgart, Germany as an intern in 2005 and she was a research assistant in 2007 at the Department of Electrical \& Electronics Engineering, The University of Melbourne, Australia. Since July 2007, she has been an Assistant Professor at the Department of Industrial Design, Eindhoven University of Technology, the Netherlands, where she also served as Chair of the Theme Health Care from 2009 to 2013. Her research interests include medical monitoring system design, patient health monitoring, wireless body area networks, smart sensor systems, ambient intelligence and smart environments. She is a Senior Member of IEEE.

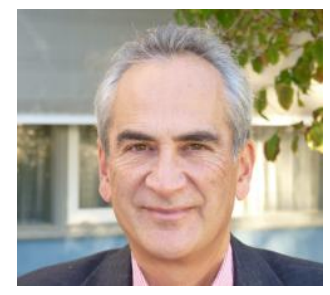

Sidarto Bambang Oetomo received his Medical Doctor degree in 1979 from the Faculty of Medicine at the University of Groningen, The Netherlands and specialized in Pediatrics, registration as Pediatrician in 1983 and subsequently in Neonatology at the same institution. He obtained his Ph.D. degree in 1988 on Treatment of surfactant deficiency in preterm infants. He was research fellow at the Perinatal Research Laboratory at Harbor UCLA, Torrance California from 1988 to '89. In 1996 he was appointed as Professor of Pediatrics and Neonatology at the University of Groningen. The focus on his research was on neonatal lung disease, surfactant treatments and inflammation. In 2003 he accepted the position of consultant in Neonatology at the Máxima Medical Center, Veldhoven, The Netherlands. He was appointed as Professor at the Faculty of Industrial Design of the Eindhoven University of Technology in 2007. His main research interest is in the field of ambient intelligence and monitoring of vital functions of newborn infants using non-obtrusive technologies. He is member of the Dutch Pediatric Society, the European Society for Pediatric Research and the American Society of Pediatric Research.

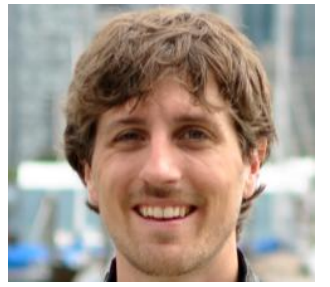

Daniel Tetteroo received his MSc. in Human Media Interaction from University of Twente, The Netherlands in 2010 and his PDEng degree on User System Interaction from Eindhoven University of Technology, The Netherlands, in 2012. He has done research on various topics, ranging from child-computer interaction and mobile interaction to design for neonatal care units. Currently, he works as a Ph.D. researcher at Eindhoven University of Technology on the application of end-user development principles in the domain of physical rehabilitation.

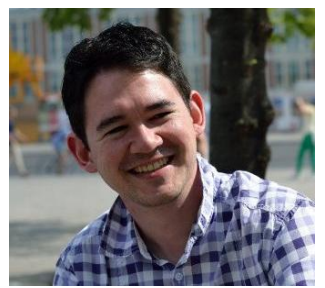

Frank Versteegh received his MSc. degree in 2010 from Leiden University where he specialized in applied cognitive psychology. He obtained his PDEng degree on User System Interaction from Eindhoven University of Technology, The Netherlands in 2012. He has done an internship at the National Aerospace Laboratory in 2008 in Amsterdam and worked on a UX research project at Alcatel-Lucent in Antwerp. His main interests are in human computer interaction, interaction design and user experience research.

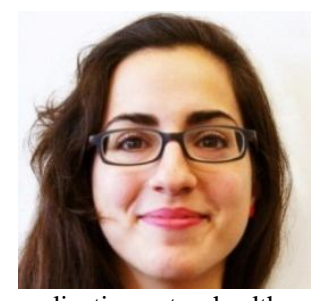

Thelxi Mamagkaki received her BSc. \& MSc. degree in Computer Engineering and Informatics in 2010 from the Polytechnic School of University of Patras, Greece. She obtained her PDEng degree on User System Interaction from Eindhoven University of Technology in the Netherlands. She has worked on designing the interaction and user experience of diverse systems, from web and mobile applications to healthcare, focusing on neonatal care and pregnancy monitoring. Her main interests are human computer interaction, interaction design and user experience.

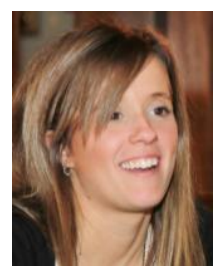

Mariana Serras Pereira is passionate about understanding human behavior, in finding and developing concrete solutions that contribute to improve people's life. As a Ph.D. researcher at Tilburg University, The Netherlands, she's focusing on how people express deception in nonverbal communication. In addition, for the last two years and half, she has worked and developed herself as user researcher and interaction designer, which allows her to apply her psychology background while learning to create product-driven solutions to improve people's life.

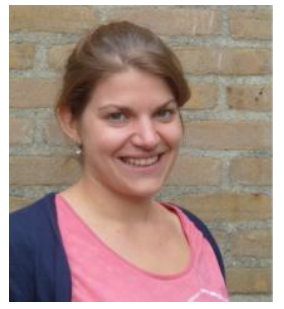

Lindy Janssen did a research internship in 2008 at the Department of Audiology and Neurootology, University Hospital Basel, Switzerland. In 2011 she received her Medical Doctor degree from the Faculty of Medicine at the University of Nijmegen, The Netherlands. She started working at the Máxima Medical Center, Veldhoven. In 2012 she worked at the Neonatal Intensive Care Unit of the Radboud UMC, Nijmegen, The Netherlands. In 2013 she got accepted for the specialization of Pediatrics. She is a member of the Dutch Pediatric Society.

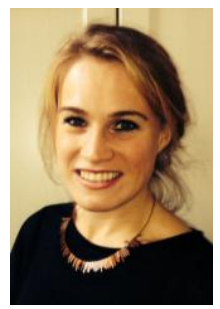

Andrea van Meurs is a medical student at the University of Utrecht, the Netherlands. She received her Bachelor medical degree in 2011 and is expected to receive her Medical Doctor degree from the Faculty of Medicine at the University of Utrecht, in 2014. In 2012 she completed her senior residency program at the Neonatal Intensive Care Unit at the Maxima Medical Centre, Veldhoven, The Netherlands. The focus on her residency program was on premature infants suffering from pain and distress during the neonatal period. Her current and future focus will be on perinatology. 